BULL. AUSTRAL. MATH. SOC.

VOL. $19(1978), 125-129$.

\title{
A result in queueing theory
}

\section{A. Ghosal}

In a single-server queueing system, subject to the queue discipline 'First come first served', the equilibrium distribution function of the waiting time of a server depends on the distribution of the random variable $(u)$ which is the difference between the service time and the inter-arrival time. If in two queueing systems $u^{\prime} s$ are equivalent in distribution, the waiting times are also equivalent in distribution (known result). It has been shown in this note that equivalence in waiting time distributions does not necessarily imply equivalence in distributions of $u^{\prime} s$. The proof is heuristic. This result has useful practical implications.

\section{Introduction}

Let us consider a single-server queueing system in which $W_{n}$ is the waiting time of $n t h$ customer, measured from his time of arrival in the queue to the time his service starts, $s_{n}$ the service time, and $t_{n}$ the time interval between arrivals of the $n$th and $(n+1)$ th customers. Let it be assumed that the queue discipline is 'First come first served'. If the waiting room has an infinite capacity and there is no constraint on the waiting time of a customer, then $W_{n+1}$ is related to $W_{n}$ in the following manner :

$$
w_{n+1}=\left(w_{n}+u_{n}\right)^{+}
$$

Received 11 July 1978. The author thanks Dr A. Ramachandran, Director-General, Council of Scientific and Industrial Research, New Delhi, for support to studies in Statistics and Operational Research. 
where $u_{n}=s_{n}-t_{n}$. If both $s_{n}$ and $t_{n}$ are random variables, $u_{n}$ is also a random variable, and the process $W$, given by (1.1), is an embedded Markov process (Lindley [3]). Let $F(y)=\operatorname{prob}\left(W_{n} \leq y\right)$ be the equilibrium distribution function of $W$, and $G(x)=\operatorname{prob}\left(u_{n} \leq x\right)$ the distribution function of $u$; then we can derive the distribution function of $W$ from that of $u$ from the following integral equation [3]:

$$
F(y)=-\int_{0}^{\infty} F(t) d G(y-t), \quad y \in(0, \infty) .
$$

The above is an integral equation of Wiener-Hopf type, and it has been shown that if $G(\cdot)$ is continuous over the domain $(-\infty, \infty)$, then for a specified distribution function $G(\cdot)$, we obtain $F(y)$ in a unique manner. If, therefore, there are two queueing systems in which distribution functions of $u$ are $G_{1}(\cdot)$ and $G_{2}(\cdot)$, and distribution functions of $W$ are $F_{1}(\cdot)$ and $F_{2}(\cdot)$, then

$$
\begin{aligned}
G_{1}(x)=G_{2}(x) \text { for all } & x \in(-\infty, \infty) \\
& \text { implies } F_{1}(y)=F_{2}(y) \text { for all } y \in[0, \infty) .
\end{aligned}
$$

We state (1.3) in the form of Lemma 1.1 below and give an alternative proof.

LEMMA 1.1. $u^{(1)} \sim u^{(2)}$ implies $w^{(1)} \sim w^{(2)}$ where $u^{(i)}$ is the $u$ for the system $i, w^{(i)}$ the waiting time for the system $i(i=1,2)$, and ' $\sim$ ' implies 'equivalence in distribution functions'.

We can also prove this lemma from Smith's [4] paper. Let $U(\alpha)$ and $W(\alpha)$ be the Laplace transforms of $u$ and $W$ respectively. Then Smith derives

$$
1-U(\alpha)=\alpha^{+}(\alpha) / \varphi^{-}(\alpha)
$$

where $\varphi(\alpha)$ is analytic and free from zeros in the right half plane $r e(\alpha)>-\gamma$, and $\varphi^{-}(\alpha)$ is analytic and free from zeros in the left half plane $r e(\alpha)<\gamma$. Then he proves that

$$
W(\alpha)=\varphi^{+}(0) / \varphi^{+}(\alpha) \text {. }
$$

Let $1-U_{1}(\alpha)=\alpha \varphi_{1}^{+}(\alpha) / \varphi_{1}^{-}(\alpha), \quad 1-U_{2}(\alpha)=\alpha \varphi_{2}^{+}(\alpha) / \varphi_{2}^{-}(\alpha)$. Then 
$U_{1}(\alpha)=U_{2}(\alpha)$, if $\varphi_{1}^{+}(\alpha)=\varphi_{2}^{+}(\alpha)$ and $\varphi_{1}^{-}(\alpha)=\varphi_{2}^{-}(\alpha)$. Hence $W_{1}(\alpha)=W_{2}(\alpha)$.

\section{Basic result}

We prove the following theorem.

THEOREM 2.1. Let there be two queueing systems in which $u^{(1)}=s^{(1)}-t^{(1)}$ and $u^{(2)}=s^{(2)}-t^{(2)}$, and $w^{(1)}$ and $w^{(2)}$ are waiting times. While equivalence in distribution functions of $u^{(1)}$ and $u^{(2)}$ ensures equivalence in distribution functions of $w^{(1)}$ and $w^{(2)}$, equivalence in distribution functions of $W^{(1)}$ and $W^{(2)}$ does not necessarily ensure equivalence in distribution functions of $u^{(1)}$ and $u^{(2)}$.

Proof. The fact that in any two systems $u^{(1)} \sim u^{(2)}$ implies $W^{(1)} \sim W^{(2)}$ is proved in Lemma 1.1 .

The second part of the theorem, namely that $W^{(1)}$ and $W^{(2)}$ can be equivalent in distribution functions without $u^{(1)}$ and $u^{(2)}$ being equivalent in distribution functions is proved by citing an example. It has been proved by Smith [4] that in a GI/M/I system (with a general arrival distribution function and exponential service distribution function) the waiting time follows an exponential distribution function. From this, Ghosal ([2], Theorem 3.3 proved that for any GI/M/I system, an M/M/1 isomorph (with respect to waiting time distribution functions) exists: also the service time in two such systems are the same, but the inter-arrival distribution function is general in $G I / M / I$ (system $A_{1}$ ) and exponential in the $M / M / 1$ isomorph (system $A_{2}$ ). For adefinition of restricted isomorph see [3]: for example, if two systems have the same waiting distribution function but their idle time (of server) distributions functions are different, one is an isomorph of the other in the restricted sense.) Thus, we have $u_{1}$ (for system $A_{1}$ ) and $u_{2}$ (for system $A_{2}$ ) such that 


$$
u_{1}=s_{1}-t_{1}, u_{2}=s_{2}-t_{2}
$$

where $s_{1} \sim s_{2}$ but $t_{1}$, and $t_{2}$ are not equivalent in distribution functions. Hence $u_{1}$ and $u_{2}$ are not equivalent in distribution functions. Thus, Theorem 2.1 follows. In Section 3 below we discuss the conditions under which Theorem 2.1 is valid under more general situations.

\section{Interpretation of Theorem 2.1}

Let $W(\alpha)$ be the Laplace transform of the waiting time distribution function, and $U(\alpha)$ the Laplace transform of $U$. Then, from Lindley [3] and Ghosal ([1], equation (1.34)),

$$
W(\alpha)[U(\alpha)-1]=n^{+}(\alpha)-F^{\prime}(0),
$$

where $n^{+}(\alpha)$ is the Laplace transform of the idle-time of server (I), leaving the concentration at zero $(\operatorname{pr}(I=0)=(I-F(0)))$; $F(0)=\operatorname{prob}(W=0)=\operatorname{prob}(I>0)$. In deriving (3.1), we assume a distribution function $F^{*}(y)$ which is related to $F(y)$, the waiting time distribution function of $W$, as follows:

$$
\begin{aligned}
F^{*}(y) & =F(y) \text { on } y \in[0, \infty) \\
& =\operatorname{prob}(-I \leq y)=\operatorname{prob}(I \geq-y) \text { on } y \in(-\infty, 0) .
\end{aligned}
$$

We have

$$
\eta(\alpha)=\int_{-\infty}^{0} \exp (-\alpha y) d F^{*}(y)=1-F(0)+\eta^{+}(\alpha)
$$

In the two systems $A_{1}$ and $A_{2}$ referred to in Theorem 2.1, we get, since $W_{1} \sim W_{2}$ (so that $W_{1}(\alpha)=W_{2}(\alpha)=W(\alpha)$, and $F_{1}(0)=F_{2}(0)$ ),

$$
\left[U_{1}(\alpha)-1\right] /\left[U_{2}(\alpha)-1\right]=\left[n_{1}(\alpha)-1\right] /\left[n_{2}(\alpha)-1\right] \text {, }
$$

or

$$
\left[\eta_{1}(\alpha)-\eta_{2}(\alpha)\right]=W(\alpha)\left[U_{1}(\alpha)-U_{2}(\alpha)\right]
$$

When Theorem 2.1 holds, the relation (3.2) or (3.3) holds.

\section{Concluding remarks}

It is possible that Theorem 2.1 holds true for a large number of pairs 
of $G l / G / l$ systems. The condition for its validity is (3.2) or (3.3) which leads to possibilities of good simulation experiments. Theorem 2.1 provides a basic relation between two single-server queueing systems one of which is an isomorph of the other in the restricted sense - in other words, two are equivalent in respect of any one or two but not all of the output elements - here they are equivalent in respect of waiting time distribution functions; see [2] for more details of properties of isomorphic queues.

\section{References}

[1] A. Ghosal, Some aspects of queueing and storage systems (Lecture Notes in Operations Research and Mathematical Systems, 23. Springer-Verlag, Berlin, Heidelberg, New York, 1970).

[2] A. Ghosal, "Isomorphic queues", Bull. Austral. Math. Soc. 17 (1977), 275-289.

[3] D.V. Lindley, "The theory of queues with a single server", Proc. Cambridge Phizos. Soc. 48 (1952), 277-289.

[4] Walter M. Smith, "On the distribution of queueing times", Proc. Cambridge Philos. Soc. 49 (1953), 449-461.

Council of Scientific and Industrial Research, Rafi Marg, New Delhi, India. 